\title{
Voronoi-Based Geometry Estimator for 3D Digital Surfaces ${ }^{\star}$
}

\author{
Louis Cuel $^{1,2}$, Jacques-Olivier Lachaud ${ }^{1}$, and Boris Thibert ${ }^{2}$ \\ 1 Université de Savoie, Laboratoire LAMA, \\ Le bourget du lac, France \\ ${ }^{2}$ Université de Grenoble, Laboratoire Jean Kuntzmann, \\ Grenoble, France
}

\begin{abstract}
We propose a robust estimator of geometric quantities such as normals, curvature directions and sharp features for 3D digital surfaces. This estimator only depends on the digitisation gridstep and is defined using a digital version of the Voronoi Covariance Measure, which exploits the robust geometric information contained in the Voronoi cells. It has been proved in [1] that the Voronoi Covariance Measure is resilient to Hausdorff noise. Our main theorem explicits the conditions under which this estimator is multigrid convergent for digital data. Moreover, we determine what are the parameters which maximise the convergence speed of this estimator, when the normal vector is sought. Numerical experiments show that the digital VCM estimator reliably estimates normals, curvature directions and sharp features of 3D noisy digital shapes.
\end{abstract}

\section{Introduction}

Differential quantities estimation, surface reconstruction and sharp feature detection are motivated by a large number of applications in computer graphics, geometry processing or digital geometry.

Digital geometry estimators. The commun way to link the estimated differential quantities to the expect Euclidean one is the multigrid convergence principle: when the shape is digitized on a grid with gridstep $h$ tending to zero, the estimated quantity should converge to the expected one. In dimension 2, several multigrid convergent estimators have been introduced to approach normals [2, 3] and curvatures [3 5]. In 3D, empirical methods for normal and curvature estimation have been introduced in [6]. More recently, a convergent curvature estimator based on covariance matrix was presented in [7].

\footnotetext{
* This research has been supported in part by the ANR grants DigitalSnow ANR-11BS02-009, KIDICO ANR-2010-BLAN-0205 and TopData ANR-13-BS01-0008.
}

E. Barcucci et al. (Eds.): DGCI 2014, LNCS 8668, pp. 134-149 2014.

(C) Springer International Publishing Switzerland 2014 
Voronoi-based geometry estimation. Classical principal component analysis methods try to estimate normals by fitting a tangent plane or a higher-order polynomial (e.g. see [8]). In contrast, Voronoi-based methods try to fit the normal cones to the underlying shape, either geometrically 9] or more recently using the covariance of the Voronoi cells [1, 10]. Authors of [1] have improved the method of [10] by changing the domain of integration and the averaging process. The authors define the Voronoi Covariance Measure (VCM) of any compact sets, and show that this notion is stable under Hausdorff perturbation. Moreover, the VCM of a smooth surface encodes a part of its differential information, such as its normals and curvatures. With the stability result, one can therefore use the VCM to estimate differential quantities of a surface from a Hausdorff approximation such as a point cloud or a digital contour.

Voronoi Covariance measure background. The Voronoi covariance measure (VCM) has been introduced in [1] for normals and curvature estimations. Let $K$ be a compact subset of $\mathbb{R}^{3}$ and $d_{K}$ the distance function to $K$, i.e. the $\operatorname{map~}_{K}(x):=\min _{p \in K}\|p-x\|$. A point $p$ where the previous minimum is reached is called a projection of $x$ on $K$. Almost every point admits a single projection on $K$, thus definining a map $\mathrm{p}_{K}: \mathbb{R}^{3} \rightarrow K$ almost everywhere. The $R$-offset of $K$ is the $R$-sublevel set of $\mathrm{d}_{K}$, i.e. the set $K^{R}:=d_{K}^{-1}(]-\infty, R[)$. The VCM maps any integrable function $\chi: \mathbb{R}^{3} \rightarrow \mathbb{R}^{+}$to the matrix

$$
\mathcal{V}_{K, R}(\chi):=\int_{K^{R}}\left(x-p_{K}(x)\right)\left(x-p_{K}(x)\right)^{\mathbf{t}} \chi\left(p_{K}(x)\right) \mathrm{d} x .
$$

Remark that this definition matches the definition introduced in [1]: when $\chi$ is the indicatrix of a ball, one recovers a notion similar to the convolved $\mathrm{VCM}: \mathcal{V}_{K, R}(\chi):=\int_{K^{R} \cap p_{K}^{-1}\left(\mathcal{B}_{y}(r)\right)}\left(x-p_{K}(x)\right)\left(x-p_{K}(x)\right)^{\mathbf{t}} \mathrm{d} x$. The domain of integration $K^{R} \cap p_{K}^{-1}\left(\mathcal{B}_{y}(r)\right)$ is the offset of $\mathrm{K}$ intersected with a union of Voronoi cells (cf. Figure [1). The stability result of [1] implies that

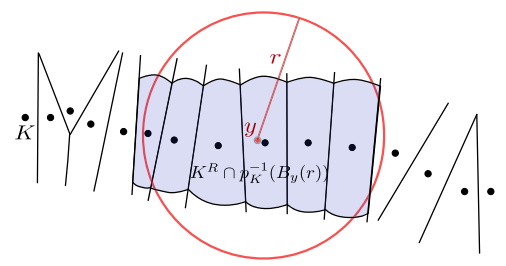

Fig. 1. VCM domain of integration 
information extracted from the covariance matrix such as normals or principal directions are stable with respect to Hausdorff perturbation.

Contributions. The contributions of the paper can be sketched as follows. First, we define the estimator of the VCM in the case of digital data, for which we prove the multigrid convergence (Sect. 2, Theorem 1). We then show that the normal direction estimator, defined as the first eigenvector of the VCM estimator, is also convergent with a speed in $O\left(h^{\frac{1}{8}}\right)$ (Sect. 3, Corollary 1). Furthermore, Theorem 2 specifies how to choose parameters $r$ and $R$ as functions of $h$ to get the convergence. Finally, we present an experimental evaluation showing that this convergence speed is closer to $O(h)$ in practice (Sect. 4). Moreover, experiments indicate that the VCM estimator can be used to estimate curvature information and sharp features in the case of digital data perturbated by Hausdorff noise.

\section{VCM on Digital Sets}

In this section, we define an estimator of the VCM in the case of 3D digital input. Theorem 1 explicits the conditions under which this estimator is multigrid convergent for digital data.

\subsection{Definition}

Let $X$ be a compact domain of $\mathbb{R}^{3}$ whose boundary is a surface of class $C^{2}$. We denote $\partial X$ the boundary of $X, X_{h}:=\operatorname{Dig}_{h}(X)=X \cap(h \mathbb{Z})^{3}$ the Gauss digitisation of $X$, and $\partial_{h} X \subset \mathbb{R}^{3}$ the set of boundary surfels of $X_{h}$. We define a digital approximation of the VCM on a subset of the point cloud : $Z_{h}=\partial_{h} X \cap h\left(\mathbb{Z}+\frac{1}{2}\right)^{3}$. For each point $x \in h\left(\mathbb{Z}+\frac{1}{2}\right)^{3}$ with $x=\left(x_{1}, x_{2}, x_{3}\right)$, we can define the voxel of center $x$ by $\operatorname{vox}(x)=$ $\left[x_{1}-\frac{1}{2} h, x_{1}+\frac{1}{2} h\right] \times\left[x_{2}-\frac{1}{2} h, x_{2}+\frac{1}{2} h\right] \times\left[x_{3}-\frac{1}{2} h, x_{3}+\frac{1}{2} h\right]$. We then define the digital VCM estimator as

$$
\widehat{\mathcal{V}}_{Z_{h}, R}(\chi):=\sum_{x \in \Omega_{h}^{R}} h^{3}\left(x-p_{Z_{h}}(x)\right)\left(x-p_{Z_{h}}(x)\right)^{t} \chi\left(p_{Z_{h}}(x)\right),
$$

where $\Omega_{h}^{R}=\left\{x \in Z_{h}^{R} \cap h\left(\mathbb{Z}+\frac{1}{2}\right)^{3}, \operatorname{vox}(x) \subset Z_{h}^{R}\right\}$ is the set of centers of voxels entirely contained in $Z_{h}^{R}$, the $R$-offset of $Z_{h}$ (see Fig. 2). Remark that the Hausdorff distance between $\partial X$ and the point cloud $Z_{h}$ used in the definition is less than $h$. 

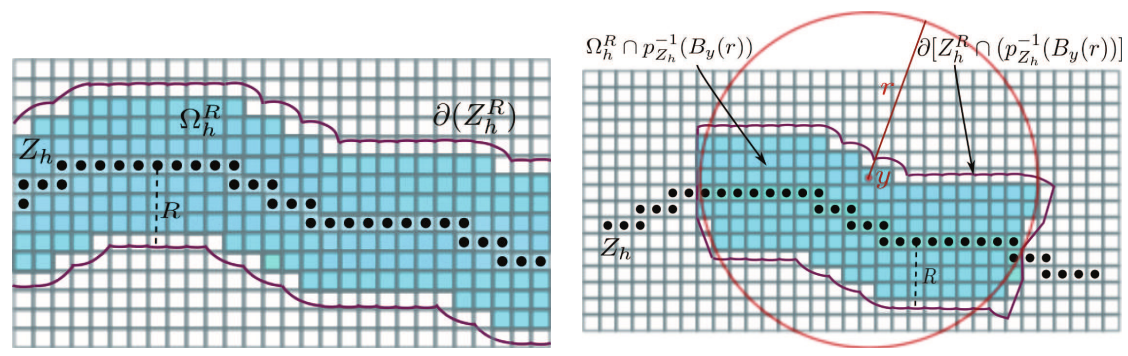

Fig. 2. Digitisation of the offset and its localisation

\subsection{Multigrid Convergence of the VCM-Estimator}

The main theoretical result of the paper is the following theorem. Roughly speaking, it quantifies the approximation of the VCM of a smooth surface by the digital VCM of its Gauss digitisation. We denote by $\|\cdot\|_{\text {op }}$ the matrix norm induced by the Euclidean metric. Given a function $f: \mathbb{R}^{n} \rightarrow$ $\mathbb{R}$, we let $\|f\|_{\infty}=\max _{x \in \mathbb{R}^{n}}|f(x)|$ and denote $\operatorname{Lip}(f)=\max _{x \neq y} \mid f(x)-$ $f(y) \mid /\|x-y\|$ its Lipschitz constant.

Theorem 1. Let $X$ be a compact domain of $\mathbb{R}^{3}$ whose boundary $\partial X$ is a $C^{2}$ surface with reach $\rho>0$. Let $R<\frac{\rho}{2}$ and $\chi: \mathbb{R}^{3} \rightarrow \mathbb{R}^{+}$be an integrable function whose support is contained in a ball of radius $r$. Then for any $h>0$ such that $h \leq \min \left(R, \frac{r}{2}, \frac{r^{2}}{32 \rho}\right)$, one has

$$
\begin{aligned}
\left\|\mathcal{V}_{\partial X, R}(\chi)-\widehat{\mathcal{V}}_{Z_{h}, R}(\chi)\right\|_{\mathrm{op}} & =O\left(\operatorname{Lip}(\chi) \times\left[\left(r^{3} R^{\frac{5}{2}}+r^{2} R^{3}+r R^{\frac{9}{2}}\right) h^{\frac{1}{2}}\right]\right. \\
& \left.+\|\chi\|_{\infty} \times\left[\left(r^{3} R^{\frac{3}{2}}+r^{2} R^{2}+r R^{\frac{7}{2}}\right) h^{\frac{1}{2}}+r^{2} R h\right]\right) .
\end{aligned}
$$

In the theorem and in the following of the text, the constant involved in the notation $O($.$) only depends on the reach of \partial X$ and on the dimension (which is three here).

For the proof of Theorem 1, we introduce the VCM of the point cloud $Z_{h}$, namely $\mathcal{V}_{Z_{h}, R}(\chi)$. By the triangle inequality, one has

$$
\left\|\mathcal{V}_{\partial X, R}(\chi)-\widehat{\mathcal{V}}_{Z_{h}, R}(\chi)\right\|_{\mathrm{op}} \leq\left\|\mathcal{V}_{\partial X, R}(\chi)-\mathcal{V}_{Z_{h}, R}(\chi)\right\|_{\mathrm{op}}+\left\|\mathcal{V}_{Z_{h}, R}(\chi)-\widehat{\mathcal{V}}_{Z_{h}, R}(\chi)\right\|_{\mathrm{op}} .
$$

In Proposition 1, we bound the second term and in Proposition 2, we bound the first term. 
Estimation of the VCM of a Point Cloud. Here and in the following of this section, $X$ is a compact domain of $\mathbb{R}^{3}$ whose boundary $\partial X$ is a $C^{2}$ surface with reach $\rho>0$. We put $R<\frac{\rho}{2}$ and $\chi: \mathbb{R}^{3} \rightarrow \mathbb{R}^{+}$is an integrable function whose support is contained in a ball $\mathcal{B}_{y}(r)$ of center $y$ and radius $r$.

Proposition 1. For any $h \leq \min \left(R, \frac{r}{2}, \frac{r^{2}}{32 \rho}\right)$, one has $\left\|\mathcal{V}_{Z_{h}, R}(\chi)-\widehat{\mathcal{V}}_{Z_{h}, R}(\chi)\right\|_{\mathrm{op}}=O\left[r^{2} R^{2}\left(\operatorname{Lip}(\chi) R+\|\chi\|_{\infty}\right) h^{\frac{1}{2}}+r^{2} R\left\|_{\chi}\right\|_{\infty} h\right]$.

Proof. Step 1: The aim of the first step is to prove that $\mathcal{V}_{Z_{h}, R}(\chi)=\int_{\operatorname{vox}\left(\Omega_{h}^{R}\right)}\left(x-p_{Z_{h}}(x)\right)\left(x-p_{Z_{h}}(x)\right)^{\mathbf{t}} \chi\left(p_{Z_{h}}(x)\right) \mathrm{d} x+R^{2}\|\chi\|_{\infty} O\left(h r^{2}\right)$. Since $\operatorname{vox}\left(\Omega_{h}^{R}\right) \subset Z_{h}^{R}$, one has

$$
\begin{aligned}
\mathcal{V}_{Z_{h}, R}(\chi) & =\int_{\operatorname{vox}\left(\Omega_{h}^{R}\right)}\left(x-p_{Z_{h}}(x)\right)\left(x-p_{Z_{h}}(x)\right)^{\mathbf{t}} \chi\left(p_{Z_{h}}(x)\right) \mathrm{d} x \\
& +\int_{Z_{h}^{R} \backslash \operatorname{vox}\left(\Omega_{h}^{R}\right)}\left(x-p_{Z_{h}}(x)\right)\left(x-p_{Z_{h}}(x)\right)^{\mathbf{t}} \chi\left(p_{Z_{h}}(x)\right) \mathrm{d} x
\end{aligned}
$$

By using the facts that $\left\|x-p_{Z_{h}}(x)\right\| \leq R, \chi$ is bounded by $\|\chi\|_{\infty}$, and the support of $\chi$ is contained in the ball $\mathcal{B}_{y}(r)$ (see Figure 2), the second term of the previous equation is bounded by

$$
R^{2} \times\|\chi\|_{\infty} \times \mathcal{H}^{3}\left(\left[Z_{h}^{R} \backslash \operatorname{vox}\left(\Omega_{h}^{R}\right)\right] \cap p_{Z_{h}}^{-1}\left(\mathcal{B}_{y}(r)\right)\right) .
$$

Now, we claim that $Z_{h}^{R} \cap p_{Z_{h}}^{-1}\left(\mathcal{B}_{y}(r)\right) \subset p_{\partial X}^{-1}\left(\mathcal{B}_{y}(2 r)\right)$. Indeed, let $x \in$ $Z_{h}^{R} \cap p_{Z_{h}}^{-1}\left(\mathcal{B}_{y}(r)\right)$. The fact that the Hausdorff distance between $Z_{h}$ and $\partial X$ is less than $h$ implies that $x \in \partial X^{R+h}$. Now, since $h \leq R$, Lemma 3 implies that $\left\|p_{\partial X}(x)-p_{Z_{h}}(x)\right\| \leq \sqrt{8 h \rho}+h$, which leads to

$$
\left\|p_{\partial X}(x)-y\right\| \leq\left\|p_{\partial X}(x)-p_{Z_{h}}(x)\right\|+\left\|p_{Z_{h}}(x)-y\right\| \leq \sqrt{8 h \rho}+h+r \leq 2 r .
$$

Now, we show that $Z_{h}^{R} \backslash \operatorname{vox}\left(\Omega_{h}^{R}\right) \subset \partial X^{R+h} \backslash \partial X^{R-(\sqrt{3}+1) h}$. Indeed, as said just before, one has $Z_{h}^{R} \subset \partial X^{R+h}$. Furthermore, if $x \in \partial X^{R-(\sqrt{3}+1) h}$, then the fact that the Hausdorff distance between $Z_{h}$ and $\partial X$ is less than $h$ implies that $x \in Z_{h}^{R-\sqrt{3} h}$. Let $c \in h\left(\mathbb{Z}+\frac{1}{2}\right)^{3}$ be the center of a voxel containing $x$. The fact that $\operatorname{diam}(\operatorname{vox}(c))=\sqrt{3} h$ implies that $\operatorname{vox}(c) \subset$ $Z_{h}^{R}$, and thus $x \in Z_{h}^{R}$. We then get $Z_{h}^{R} \backslash \operatorname{vox}\left(\Omega_{h}^{R}\right) \subset \partial X^{R+h} \backslash \partial X^{R-(\sqrt{3}+1) h}$. We finally deduce that

$$
\left[Z_{h}^{R} \backslash \operatorname{vox}\left(\Omega_{h}^{R}\right)\right] \cap p_{Z}^{-1}\left(\mathcal{B}_{y}(r)\right) \subset\left[\partial X^{R+3 h} \backslash \partial X^{R-3 h}\right] \cap p_{\partial X}^{-1}\left(\mathcal{B}_{y}(2 r)\right),
$$


whose volume is bounded by $O\left(h r^{2}\right)$ by Proposition 3, which allows us to conclude.

Step 2: We then have to bound the remaining term

$$
\Delta=\int_{\operatorname{vox}\left(\Omega_{h}^{R}\right)}\left(x-p_{Z_{h}}(x)\right)\left(x-p_{Z_{h}}(x)\right)^{\mathbf{t}} \chi\left(p_{Z_{h}}(x)\right) \mathrm{d} x-\widehat{\mathcal{V}}_{Z_{h}, R}(\chi) .
$$

By decomposing $\Delta$ over all the voxels of $\operatorname{vox}\left(\Omega_{h}^{R}\right)$, one has

$$
\begin{aligned}
\Delta=\sum_{c \in \Omega_{h}^{R}} \int_{\operatorname{vox}(c)}\left[\left(x-p_{Z_{h}}(x)\right)\left(x-p_{Z_{h}}(x)\right)^{\mathbf{t}} \chi\left(p_{Z_{h}}(x)\right)\right. \\
\left.-\left(c-p_{Z_{h}}(c)\right)\left(c-p_{Z_{h}}(c)\right)^{\mathbf{t}} \chi\left(p_{Z_{h}}(c)\right)\right] \mathrm{d} x
\end{aligned}
$$

As in Step 1, we can localise the calculation around the support of $\chi$ and we introduce the set of centers $D=\Omega_{h}^{R} \cap p_{\partial X}^{-1}\left(\mathcal{B}_{y}(2 r)\right)$. Using the relation $\chi\left(p_{Z_{h}}(c)\right)=\chi\left(p_{Z_{h}}(c)\right)+\chi\left(p_{Z_{h}}(x)\right)-\chi\left(p_{Z_{h}}(x)\right)$, one gets $\Delta=\Delta_{1}+\Delta_{2}$, where

$$
\begin{aligned}
& \Delta_{1}=\sum_{c \in D} \int_{\operatorname{vox}(c)}\left(x-p_{Z_{h}}(x)\right)\left(x-p_{Z_{h}}(x)\right)^{\mathbf{t}}\left[\chi\left(p_{Z_{h}}(x)\right)-\chi\left(p_{Z_{h}}(c)\right)\right] \mathrm{d} x \\
& \Delta_{2}=\sum_{c \in D} \int_{\operatorname{vox}(c)}\left[\left(x-p_{Z_{h}}(x)\right)\left(x-p_{Z_{h}}(x)\right)^{\mathbf{t}}-\left(c-p_{Z_{h}}(c)\right)\left(c-p_{Z_{h}}(c)\right)^{\mathbf{t}}\right] \chi\left(p_{Z_{h}}(c)\right)
\end{aligned}
$$

We are now going to bound $\Delta_{1}$ and $\Delta_{2}$. One has

$$
\left\|\Delta_{1}\right\|_{\mathrm{op}} \leq \sum_{c \in D} \int_{\operatorname{vox}(c)}\left\|x-p_{Z_{h}}(x)\right\|\left\|x-p_{Z_{h}}(x)^{\mathbf{t}}\right\|\left\|\chi\left(p_{Z_{h}}(x)\right)-\chi\left(p_{Z_{h}}(c)\right)\right\| \mathrm{d} x .
$$

For all $c \in D$ and $x \in \operatorname{vox}(c)$, we have $\|x-c\| \leq \frac{\sqrt{3}}{2} h$. Furthermore, by definition of $\Omega_{h}^{R}$, we have that $x$ and $c$ belong to $Z_{h}^{R} \subset \partial X^{R+h}$. Then, since $h \leq R \leq \frac{\rho}{2}$, Proposition 4 implies $\left\|p_{Z_{h}}(x)-p_{Z_{h}}(c)\right\|=O\left(h^{\frac{1}{2}}\right)$ and then $\left\|\chi\left(p_{Z_{h}}(x)\right)-\chi\left(p_{Z_{h}}(c)\right)\right\|=\operatorname{Lip}(\chi) O\left(h^{\frac{1}{2}}\right)$. Using the fact that $\left\|x-p_{Z_{h}}(x)\right\| \leq R$, one has

$$
\left\|\Delta_{1}\right\|_{\text {op }}=\operatorname{Vol}(\operatorname{vox}(D)) \times R^{2} \times \operatorname{Lip}(\chi) \times O\left(h^{\frac{1}{2}}\right) .
$$

Since $\operatorname{vox}(D) \subset Z_{h}^{R} \cap p_{\partial X}^{-1}\left(\mathcal{B}_{y}(2 r)\right) \subset \partial X^{R+h} \cap p_{\partial X}^{-1}\left(\mathcal{B}_{y}(2 r)\right)$ and $h \leq R$, Proposition 3 implies that $\operatorname{Vol}(\operatorname{vox}(D))=O\left(r^{2} R\right)$. Finally $\left\|\Delta_{1}\right\|_{\mathrm{op}}=$ $\operatorname{Lip}(\chi) \times O\left(r^{2} R^{3} h^{\frac{1}{2}}\right)$.

Similarly, let us bound $\left\|\Delta_{2}\right\|_{\text {op }}$. We put $u=(x-c), v=c-p_{Z_{h}}(c)$ and $w=p_{Z_{h}}(c)-p_{Z_{h}}(x)$. We can write $x-p_{Z_{h}}(x)=u+v+w$, and we get

$$
\Delta_{2}=\sum_{c \in D}\left[\int_{\operatorname{vox}(c)}\left[(u+v+w)(u+v+w)^{t}-v v^{t}\right] \chi\left(p_{Z_{h}}(c)\right)\right] .
$$


From $\|u\| \leq h,\|v\| \leq R$ and $\|w\|=O\left(h^{\frac{1}{2}}\right)$, we bound the integrand by $O\left(\|\chi\|_{\infty}\left(R h^{\frac{1}{2}}+h\right)\right)$. From $\operatorname{Vol}(\operatorname{vox}(D))=O\left(r^{2} R\right)$, one has $\left\|\Delta_{2}\right\|_{\text {op }}=$ $O\left(\|\chi\|_{\infty}\left(R^{2} r^{2} h^{\frac{1}{2}}+r^{2} R h\right)\right)$.

Stability of the VCM. It is known that the VCM is stable. More precisely, Theorem 5.1 of [1] states that $\left\|\mathcal{V}_{\partial X, R}\left(\chi_{r}\right)-\mathcal{V}_{Z_{h}, R}\left(\chi_{r}\right)\right\|_{\text {op }}=$ $O\left(h^{\frac{1}{2}}\right)$. However, the constant involved in $O\left(h^{\frac{1}{2}}\right)$ depends on the whole surface $\partial X$. We provide here a more precise constant involving only local estimations, $r$ and $R$. The proof is very similar to the one of [1], except that we localise the calculation of the integral. It is given in Appendix.

Proposition 2. For any $h \leq R$ such that $\sqrt{8 h \rho}+h \leq r$, one has

$$
\begin{aligned}
& \left\|\mathcal{V}_{\partial X, R}\left(\chi_{r}\right)-\mathcal{V}_{Z_{h}, R}\left(\chi_{r}\right)\right\|_{\mathrm{op}} \\
& =O\left(\operatorname{Lip}(\chi) \times\left[\left(r^{3} R^{\frac{5}{2}}+r^{2} R^{\frac{7}{2}}+r R^{\frac{9}{2}}\right) h^{\frac{1}{2}}\right]+\|\chi\|_{\infty} \times\left[\left(r^{3} R^{\frac{3}{2}}+r^{2} R^{\frac{5}{2}}+r R^{\frac{7}{2}}\right) h^{\frac{1}{2}}\right]\right) .
\end{aligned}
$$

End of proof of Theorem 1, Let $h \leq \min \left(R, \frac{r}{2}, \frac{r^{2}}{32 \rho}\right)$. The assumption $h \leq \frac{r^{2}}{32 \rho}$ implies that $\sqrt{8 h \rho}+h \leq r$. Thus we can apply Proposition 1 and Proposition 2 .

\section{Multigrid Convergence of the Normal Estimator}

Let $X$ be a compact domain of $\mathbb{R}^{3}$ whose boundary $\partial X$ is a surface of class $C^{2}$. We now want to estimate the normal, denoted by $n\left(p_{0}\right)$, of $\partial X$ at a point $p_{0}$ from its Gauss digitisation. We define the normal estimator by applying the digital VCM on a Lipschitz function that approaches the indicatrix of the ball $\mathcal{B}_{p_{0}}(r)$.

Definition 1. The normal estimator $\widehat{n}_{r, R}\left(p_{0}\right)$ is the unit eigenvector associated to the largest eigenvalue of $\widehat{\mathcal{V}}_{Z_{h}, R}\left(\chi_{r}\right)$, where $\chi_{r}$ is a Lipschitz function that is: equal to 1 on $\mathcal{B}_{p_{0}}(r)$, equal to $1-\left(\left\|x-p_{0}\right\|-r\right) / r^{\frac{3}{2}}$ on $\mathcal{B}_{p_{0}}\left(r+r^{\frac{3}{2}}\right) \backslash \mathcal{B}_{p_{0}}(r)$, and equal to 0 elsewhere.

Remark that the normal estimator is defined only up to the sign. The following theorem gives an error estimation between $\pm \widehat{n}_{r, R}\left(p_{0}\right)$ and $n\left(p_{0}\right)$.

Theorem 2. Let $X$ be a compact domain of $\mathbb{R}^{3}$ whose boundary $\partial X$ is a $C^{2}$ surface with reach $\rho>0$. Let $R<\frac{\rho}{2}$. Then for any $h>0$ such that $h \leq \min \left(R, \frac{r}{2}, \frac{r^{2}}{32 \rho}\right)$, the angle between the lines spanned by $\widehat{n}_{r, R}\left(p_{0}\right)$ and $n\left(p_{0}\right)$ satisfies 
$\left\langle\widehat{n}_{r, R}\left(p_{0}\right), n\left(p_{0}\right)\right\rangle=O\left(\left(r R^{-\frac{3}{2}}+R^{-1}+r^{-\frac{1}{2}} R^{-\frac{1}{2}}+r^{-\frac{3}{2}}+r^{-\frac{5}{2}} R^{\frac{3}{2}}\right) h^{\frac{1}{2}}+R^{-2} h+r^{\frac{1}{2}}+R^{2}\right)$.

The following corollary is a direct consequence.

Corollary 1. Let $X$ be a compact domain of $\mathbb{R}^{3}$ whose boundary $\partial X$ is $a C^{2}$ surface with reach $\rho>0$. Let $a, b \in \mathbb{R}^{+}, r=a h^{\frac{1}{4}}$ and $R=b h^{\frac{1}{4}}$. Then for any $h>0$ small enough, one has

$$
\left\langle\widehat{n}_{r, R}\left(p_{0}\right), n\left(p_{0}\right)\right\rangle=O\left(h^{\frac{1}{8}}\right) .
$$

Proof of Theorem Q. We introduce the normalized VCM $\widehat{N}_{r, R}\left(p_{0}\right)=$ $\frac{3}{2 \pi r^{2} R^{3}} \widehat{\mathcal{V}}_{Z_{h}, R}\left(\chi_{r}\right)$. From Davis-Kahan $\sin (\theta)$ Theorem [11], up to the sign of $\pm \widehat{n}_{r, R}\left(p_{0}\right)$, one has

$$
\left\|\widehat{n}_{r, R}\left(p_{0}\right)-n\left(p_{0}\right)\right\| \leq 2\left\|\widehat{N}_{r, R}\left(p_{0}\right)-n\left(p_{0}\right) n\left(p_{0}\right)^{t}\right\|_{\mathrm{op}} .
$$

It is therefore sufficient to bound the right hand side. The triangle inequality gives

$$
\begin{aligned}
\left\|\widehat{N}_{r, R}\left(p_{0}\right)-n\left(p_{0}\right) n\left(p_{0}\right)^{t}\right\|_{\mathrm{op}} & \leq \frac{3}{2 \pi R^{3} r^{2}}\left\|\widehat{\mathcal{V}}_{Z_{h}, R}\left(\chi_{r}\right)-\mathcal{V}_{\partial X, R}\left(\chi_{r}\right)\right\|_{\mathrm{op}} \\
& +\frac{3}{2 \pi R^{3} r^{2}}\left\|\mathcal{V}_{\partial X, R}\left(\chi_{r}\right)-\mathcal{V}_{\partial X, R}\left(\mathbf{1}_{\mathcal{B}_{p_{0}}(r)}\right)\right\|_{\mathrm{op}} \\
& +\left\|\frac{3}{2 \pi R^{3} r^{2}} \mathcal{V}_{\partial X, R}\left(\mathbf{1}_{\mathcal{B}_{p_{0}}(r)}\right)-n\left(p_{0}\right) n\left(p_{0}\right)^{t}\right\|_{\mathrm{op}} .
\end{aligned}
$$

The proof of the theorem relies on Theorem 1, that controls the first term, and on the two following lemmas.

Lemma 1. Under the assumption of Theorem 2, we have

$$
\frac{3}{2 \pi r^{2} R^{3}}\left\|\mathcal{V}_{\partial X, R}\left(\chi_{r}\right)-\mathcal{V}_{\partial X, R}\left(\mathbf{1}_{\mathcal{B}_{p_{0}}(r)}\right)\right\|_{\mathrm{op}}=O\left(r^{\frac{1}{2}}\right) .
$$

Proof. Since $\chi_{r}=\mathbf{1}_{\mathcal{B}_{p_{0}}(r)}$ on the ball $\mathcal{B}_{p_{0}}(r)$, by using similar arguments as previously, one has

$\left\|\mathcal{V}_{\partial X, R}\left(\chi_{r}\right)-\mathcal{V}_{\partial X, R}\left(\mathbf{1}_{\mathcal{B}_{p_{0}}(r)}\right)\right\|_{\mathrm{op}} \leq \operatorname{Vol}\left(\partial X^{R} \cap\left[p_{\partial X}^{-1}\left(\mathcal{B}_{y}\left(r+r^{\frac{3}{2}}\right)\right) \backslash p_{\partial X}^{-1}\left(\mathcal{B}_{y}(r)\right)\right]\right) \times R^{2}$.

Proposition 3 implies that the volume $\operatorname{Vol}\left(\partial X^{R} \cap\left[p_{\partial X}^{-1}\left(\mathcal{B}_{y}\left(r+r^{\frac{3}{2}}\right)\right) \backslash p_{\partial X}^{-1}\left(\mathcal{B}_{y}(r)\right)\right]\right)$ is less than $4 R \times \operatorname{Area}\left(\mathcal{B}_{y}\left(r+r^{\frac{3}{2}}\right) \backslash \mathcal{B}_{y}(r)\right)$. The fact that this area is bounded by $O\left(r^{\frac{5}{2}}\right)$ allows to conclude. 
Lemma 2. Under the assumption of Theorem [, we have

$$
\left\|\frac{3}{2 \pi R^{3} r^{2}} \mathcal{V}_{\partial X, R}\left(\mathbf{1}_{\mathcal{B}_{p_{0}}(r)}\right)-n\left(p_{0}\right) n\left(p_{0}\right)^{t}\right\|_{\mathrm{op}}=O\left(r+R^{2}\right)
$$

Proof. We have the following relation (see Theorem 1 of [12])

$$
\mathcal{V}_{\partial X, R}\left(\mathbf{1}_{\mathcal{B}_{p_{0}}(r)}\right)=\frac{2}{3} R^{3}\left[1+O\left(R^{2}\right)\right] \int_{p \in \mathcal{B}_{p_{0}}(r) \cap S} n(p) n(p)^{t} \mathrm{~d} p .
$$

By the mean value theorem applied to the normal to $\partial X$, one has

$$
\left\|n(p)-n\left(p_{0}\right)\right\| \leq \sup _{q \in S}\|D n(q)\|_{o p} l_{p, p_{0}},
$$

where $l_{p, p_{0}}$ is the length of a geodesic joining $p$ and $p_{0}$. Since the chord $\left(p p_{0}\right)$ belongs to the offset $\partial X^{R}$, where $R<\rho$, we have $l_{p, p_{0}}=O(\| p-$ $p_{0} \|$ ) (see [13] for example). Therefore $\left\|n(p)-n\left(p_{0}\right)\right\|=O(r)$ and thus $n(p) n(p)^{t}-n\left(p_{0}\right) n\left(p_{0}\right)^{t}=O(r)$. Consequently

$$
\int_{p \in \mathcal{B}_{p_{0}}(r) \cap S} n(p) n(p)^{t} \mathrm{~d} p=\operatorname{Area}\left(\mathcal{B}_{p_{0}}(r) \cap S\right) n\left(p_{0}\right) n\left(p_{0}\right)^{t}+\operatorname{Area}\left(\mathcal{B}_{p_{0}}(r) \cap S\right) O(r) .
$$

Combining with Eq. (2), we have

$$
\frac{3}{2 R^{3} \operatorname{Area}\left(\mathcal{B}_{p_{0}}(r) \cap S\right)} \mathcal{V}_{\partial X, R}\left(\mathbf{1}_{\mathcal{B}_{p_{0}}(r)}\right)=\left[1+O\left(R^{2}\right)\right] \times\left(n\left(p_{0}\right) n\left(p_{0}\right)^{t}+O(r)\right) .
$$

We conclude by using the fact that $\operatorname{Area}\left(\mathcal{B}_{p_{0}}(r) \cap S\right)$ is equivalent to $\pi r^{2}$.

\section{Experiments}

We evaluate experimentally the multigrid convergence, the accuracy and robustness to Hausdorff noise of our normal estimator, and also its ability to detect features.

The first series of experiments analyzes the convergence of the normal estimation by VCM toward the true normal of the shape boundary $\partial X$. The shape "torus" is a torus of great radius 6 and small radius 2, and the shape "ellipsoid" is an ellipsoid of half-axes $\sqrt{90}, \sqrt{45}$ and $\sqrt{45}$. We measure the absolute angle error with $\epsilon(p)=\frac{180}{\pi} \cos ^{-1}(\hat{n}(p) \cdot n(p))$ for every pointel $p \in Z_{h}$ of the digitized shape with several normalized norms:

$$
l_{1}(\epsilon) \stackrel{\text { def }}{=} \frac{1}{\operatorname{Card}\left(Z_{h}\right)} \sum_{p \in Z_{h}} \epsilon(p), \quad l_{\infty}(\epsilon) \stackrel{\text { def }}{=} \sup _{p \in Z_{h}} \epsilon(p) .
$$



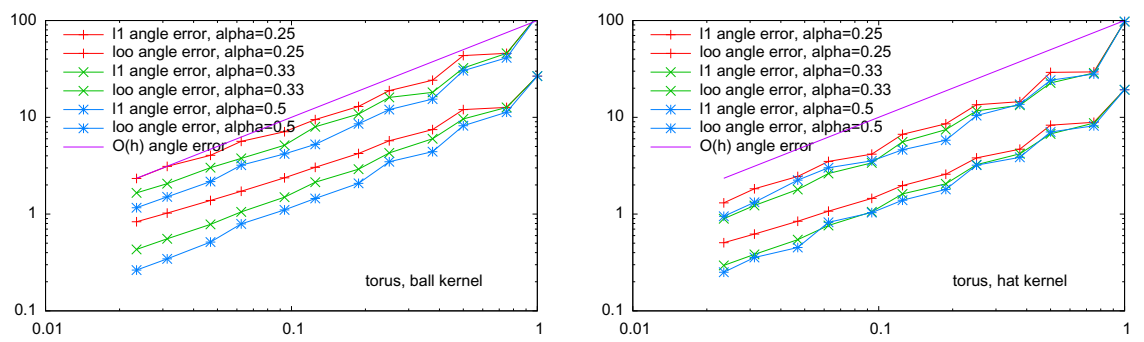

Fig. 3. Multigrid convergence of angle error of normal estimator (in degree). Abscissa is the gridstep $\mathrm{h}$. Tests are run on torus shape for three kernel radii $\left(R=r=3 h^{\alpha}\right.$ for $\left.\alpha \in\left\{\frac{1}{4}, \frac{1}{3}, \frac{1}{2}\right\}\right)$, two norms $\left(l_{1}, l_{\infty}\right)$ : (left) kernel ball function $\chi_{r}^{0}$, (right) kernel hat function $\chi_{r}^{1}$.

In experiments we tried several kernel functions $\chi_{r}$ and we display results for two of them: the "ball" kernel $\chi_{p_{0}, r}^{0}(x)=1$ if $\left\|x-p_{0}\right\| \leq r, 0$ otherwise; the "hat" kernel $\chi_{p_{0}, r}^{1}(x)=1-\left\|x-p_{0}\right\| / r$ if $\left\|x-p_{0}\right\| \leq r, 0$ otherwise. Figure 3 displays the norms of the estimation angle error in degrees, for finer and finer digitization steps. Corollary 1 predicts the multigrid convergence of the estimator when $r=a h^{\frac{1}{4}}$ and $R=b h^{\frac{1}{4}}$ at a rate in $O\left(h^{\frac{1}{8}}\right)$. We observe the convergence of the estimator for parameters $R=r=3 h^{\frac{1}{4}}, R=r=3 h^{\frac{1}{3}}, R=r=3 h^{\frac{1}{2}}$, at an almost linear rate $O(h)$, for all norms. More experiments show that the most accurate results are obtained for $\alpha \in\left[\frac{1}{3}, \frac{1}{2}\right]$ if $R=r=a h^{\alpha}$. Note that the kernel function has not a great impact on normal estimates, as long as it has a measure comparable to the ball kernel.

We perturbate the shape "torus" with a Kanungo noise model of parameter $p=0.25$ (the number $p^{d}$ is the probability that a voxel at digital distance $d$ from the boundary $\partial X$ is flipped inside/out). This is not exactly a Hausdorff perturbation but most perturbations lie in a band of size $2 h /(1-p)$. Figure 4 shows that the normal is still convergent for all norms. Again convergence speed is experimentally closer to $O\left(h^{\frac{2}{3}}\right)$, much better than the proved $O\left(h^{\frac{1}{8}}\right)$.

We then assess the visual quality of the estimators on several shapes, by rendering the digital surfels according to their estimated normals. First of all, Figure 5 displays normal estimation results on a noisy "torus" shape perturbated with a strong Kanungo noise of parameter $p=0.5$. Then, Figure 6 displays the visual improvement of using normals computed by the VCM estimator. In particular, comparing Fig $[\mathrm{b}$ and Fig $[6$ s shows that convolving Voronoi cell geometry is much more precise than convolving only surfel geometry. Furthermore, we have tested our estimator on many classical digital geometry shapes (see Figure 7). 

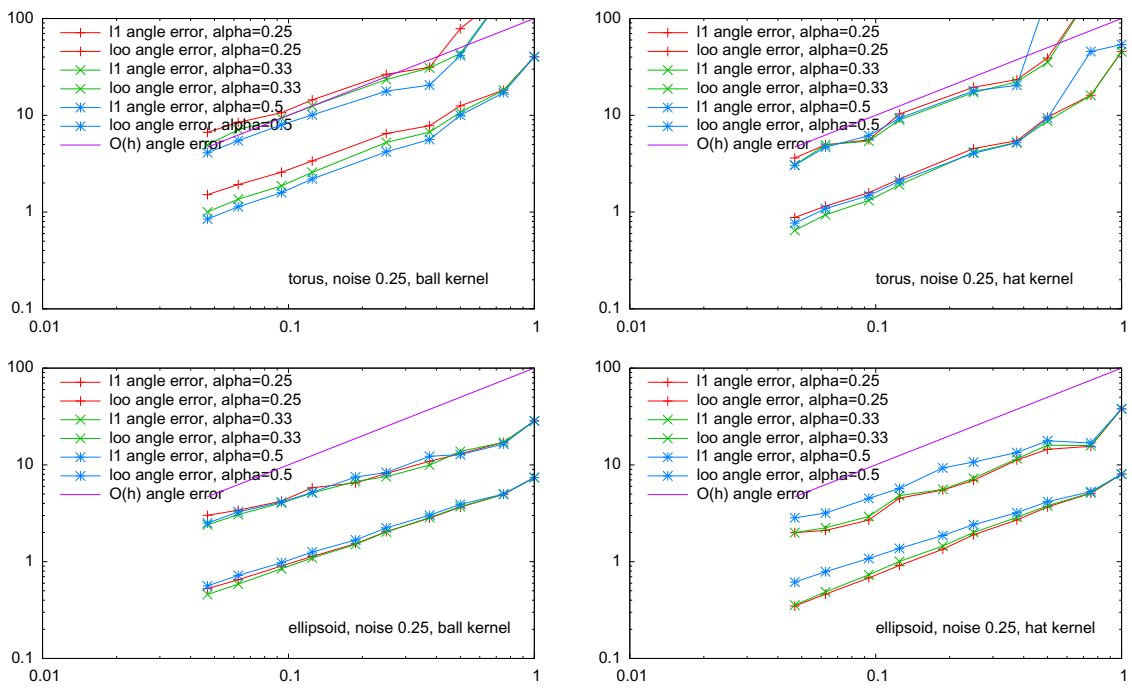

Fig. 4. Multigrid convergence of angle error of normal estimator (in degree) on a noisy shape. Abscissa is the gridstep h. Tests are run on "torus" shape (upper row) and on "ellipsoid" shape (lower row), perturbated by a Kanungo noise of parameter 0.25 , for three kernel radii $\left(R=r=3 h^{\alpha}\right.$ for $\left.\alpha \in\left\{\frac{1}{4}, \frac{1}{3}, \frac{1}{2}\right\}\right)$, two norms $\left(l_{1}, l_{\infty}\right)$ : (left) kernel ball function $\chi_{r}^{0}$, (right) kernel hat function $\chi_{r}^{1}$.

Our VCM estimator is a matrix and carries also curvature information along other eigendirections. Mérigot et al. [1] proposed to detect sharp features by using the three eigenvalues $l_{1}, l_{2}, l_{3}$ of the VCM as follows: if $l_{1} \geq l_{2} \geq l_{3}$, compute $l_{2} /\left(l_{1}+l_{2}+l_{3}\right)$ and mark the point as sharp if this value exceeds a threshold $T$. Figure 8 shows such sharp features detection on the "bunny" dataset at many different scales, with $T=0.1$ for all datasets (it corresponds to an angle of $\approx 25^{\circ}$ ). This shows that the VCM information is geometrically stable and essentially scale-invariant. To conclude, we list below some information on computation times. This estimator has been implemented using the DGtal library [14], and will soon be freely available in it.

\begin{tabular}{|cccccc|}
\hline Image & \multicolumn{4}{c|}{ size $\#$ surfels $(R, r)$} & $\chi_{r}$-VCM comput. Orienting normals \\
\hline "Al" & $150^{3}$ & 48017 & $(30,3)$ & $0.73 \mathrm{~s}$ & $0.88 \mathrm{~s}$ \\
"rcruiser" & $250^{3}$ & 66543 & $(30,3)$ & $1.26 \mathrm{~s}$ & $0.99 \mathrm{~s}$ \\
"bunny" & $516^{3}$ & 933886 & $(30,5)$ & $30.1 \mathrm{~s}$ & $15.9 \mathrm{~s}$ \\
"Dig. Snow" $512^{3}$ & 3035307 & $(30,5)$ & $82.1 \mathrm{~s}$ & $53.6 \mathrm{~s}$ \\
\hline
\end{tabular}



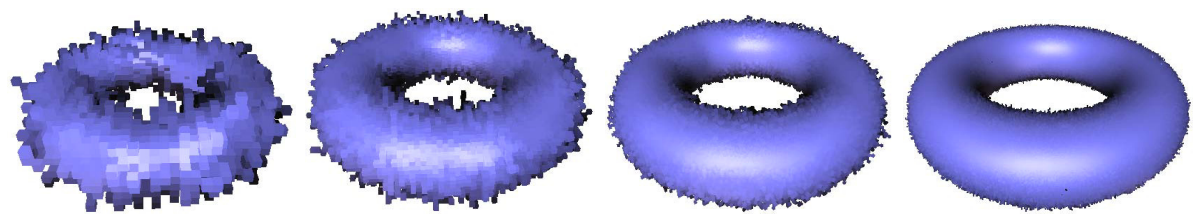

Fig. 5. Visual result of the normal estimation on the "torus" shape perturbated with a strong Kanungo noise $(p=0.5)$ for gridsteps from left to right $h=$ $0.5,0.25,0.125,0.0626$

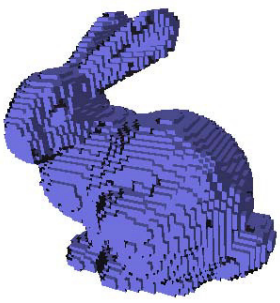

(a)

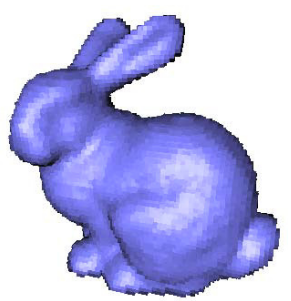

(b)

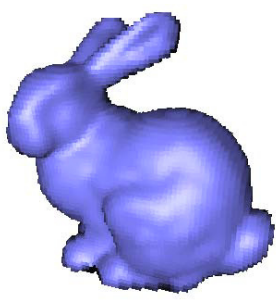

(c)

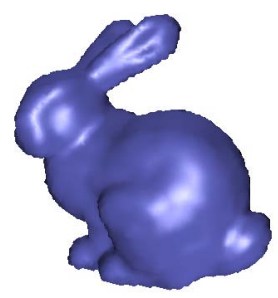

(d)

Fig. 6. Visual aspect of normal estimation on "bunny66" for $r=3$ : (a) trivial normals, (b) normals by $\chi_{r}^{1}$ convolution of trivial normals with flat shading, (c) $\chi_{r}^{1}$-VCM normals with flat shading, (d) $\chi_{r}^{1}$-VCM normals with Gouraud shading
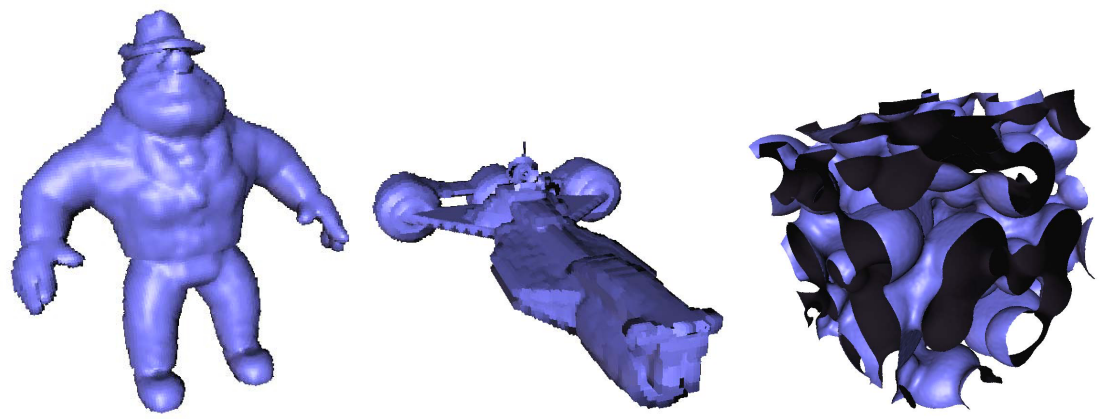

Fig. 7. Visual aspect of normal estimation on classical digital data structures: "Al" $150^{3}$, "Republic cruiser" $250^{3}$, "Digital snow" $512^{3}$ 

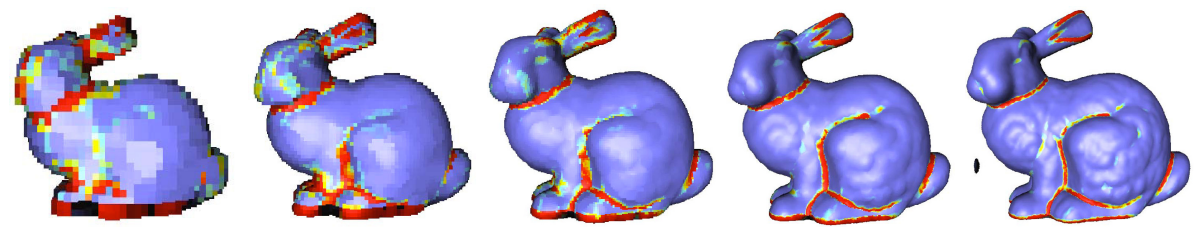

Fig. 8. Sharp feature detection on "bunny" dataset at increasing resolutions $(R=30$, $T=0.1$ ): color is metallic blue when value is in $\left[0, \frac{2}{3} T\right]$, then goes through cyan and yellow in $] \frac{2}{3} T, T[$, till red in $[T,+\infty[$

\section{Conclusion}

We have presented new stable geometry estimators for digital data, one approaching the Voronoi Covariance Measure and the other approaching the normal vector field. We have shown under which conditions they are multigrid convergent and provided formulas to determine their parameters $R$ and $r$ as a function of the gridstep $h$. Experiments have confirmed both the accuracy and the stability of our estimators. In future works, we plan to compare numerically our estimator with other discrete normal estimators (e.g. integral invariants [7], jets [15]) and also to perform a finer multigrid analysis to get a better theoretical bound on the error.

\section{References}

1. Mérigot, Q., Ovsjanikov, M., Guibas, L.: Voronoi-based curvature and feature estimation from point clouds. IEEE Transactions on Visualization and Computer Graphics 17(6), 743-756 (2011)

2. de Vieilleville, F., Lachaud, J.O.: Comparison and improvement of tangent estimators on digital curves. Pattern Recognition (2008)

3. Provot, L., Gérard, Y.: Estimation of the derivatives of a digital function with a convergent bounded error. In: Debled-Rennesson, I., Domenjoud, E., Kerautret, B., Even, P. (eds.) DGCI 2011. LNCS, vol. 6607, pp. 284-295. Springer, Heidelberg (2011)

4. Kerautret, B., Lachaud, J.O.: Curvature estimation along noisy digital contours by approximate global optimization. Pattern Recognition (2009)

5. Roussillon, T., Lachaud, J.-O.: Accurate curvature estimation along digital contours with maximal digital circular arcs. In: Aggarwal, J.K., Barneva, R.P., Brimkov, V.E., Koroutchev, K.N., Korutcheva, E.R. (eds.) IWCIA 2011. LNCS, vol. 6636, pp. 43-55. Springer, Heidelberg (2011)

6. Fourey, S., Malgouyres, R.: Normals and curvature estimation for digital surfaces based on convolutions. In: Coeurjolly, D., Sivignon, I., Tougne, L., Dupont, F. (eds.) DGCI 2008. LNCS, vol. 4992, pp. 287-298. Springer, Heidelberg (2008)

7. Coeurjolly, D., Lachaud, J.-O., Levallois, J.: Integral based curvature estimators in digital geometry. In: Gonzalez-Diaz, R., Jimenez, M.-J., Medrano, B. (eds.) DGCI 2013. LNCS, vol. 7749, pp. 215-227. Springer, Heidelberg (2013) 
8. Cazals, F., Pouget, M.: Estimating differential quantities using polynomial fitting of osculating jets. Computer Aided Geometric Design 22(2), 121-146 (2005)

9. Amenta, N., Bern, M.: Surface reconstruction by voronoi filtering. Discrete \& Computational Geometry 22(4), 481-504 (1999)

10. Alliez, P., Cohen-Steiner, D., Tong, Y., Desbrun, M.: Voronoi-based variational reconstruction of unoriented point sets. In: Symposium on Geometry Processing (2007)

11. Davis, C.: The rotation of eigenvectors by a perturbation. Journal of Mathematical Analysis and Applications (1963)

12. Mérigot, Q.: Détection de structure géométrique dans les nuages de points. $\mathrm{PhD}$ thesis, Université Nice Sophia Antipolis (December 2009)

13. Morvan, J.M., Thibert, B.: Approximation of the normal vector field and the area of a smooth surface. Discrete \& Computational Geometry 32(3), 383-400 (2004)

14. DGtal: Digital geometry tools and algorithms library, http://libdgtal.org

15. Cazals, F., Pouget, M.: Estimating differential quantities using polynomial fitting of osculating jets. Computer Aided Geometric Design 22(2), 121-146 (2005)

16. Weyl, H.: On the volume of tubes. American Journal of Mathematics, 461-472 (1939)

17. Federer, H.: Curvature measures. Trans. Amer. Math. Soc. 93(3), 418-491 (1959)

18. Cuel, L., Lachaud, J.-O., Thibert, B.: Voronoi-based geometry estimator for 3D digital surfaces. Technical report hal-00990169 (May 2014)

\section{Appendix}

We give here all the sketchs of the proofs to be self-content. Proposition 3 is classical and follows from the well-known tube formula for smooth surfaces [16]. Proposition 4 states that the projection map $p_{K}$ onto a set $K$ that is close to a smooth surface $S$ behaves like the projection map $p_{S}$. It relies on classical properties of the projection map onto a set with positive reach. The proof of Proposition 2 is similar to the proof of Theorem 5.1 of [1], except that the calculations are done locally.

\subsection{Hausdorff Measure of Offsets}

Proposition 3. Let $S \subset \mathbb{R}^{3}$ be a surface of class $C^{2}$ with reach $\rho>0$. Let $R>0$ and $\varepsilon>0$ be such that $R+\varepsilon<\frac{\rho}{2}$. Then for any ball $\mathcal{B}$ of radius $r$, one has:

a) $\operatorname{Vol}\left(S^{R} \cap p_{S}^{-1}(\mathcal{B} \cap S)\right)=O\left(R r^{2}\right)$.

b) $\operatorname{Vol}\left(\left(S^{R+\varepsilon} \backslash S^{R-\varepsilon}\right) \cap p_{S}^{-1}(\mathcal{B} \cap S)\right)=O\left(\varepsilon r^{2}\right)$.

c) Area $\left(\partial\left[S^{R} \cap p_{S}^{-1}(\mathcal{B} \cap S)\right]\right)=O\left(R r+r^{2}\right)$,

where the notation $O$ involves a constant that only depends on the reach $\rho$. 
Proof. The proof is based on the tube formula for surfaces of class $C^{2}$ [16, 17]. One has

$$
\operatorname{Vol}\left(S^{R} \cap p_{S}^{-1}(\mathcal{B} \cap S)\right)=\int_{\mathcal{B} \cap S} \int_{-R}^{R}\left(1-t \lambda_{1}(x)\right)\left(1-t \lambda_{2}(x)\right) d t d x,
$$

where $\lambda_{1}(x)$ and $\lambda_{2}(x)$ are the principal curvatures of $S$ at the point $x$. Now, since $\left|\lambda_{1}(x)\right|$ and $\left|\lambda_{2}(x)\right|$ are smaller than $\frac{1}{\rho}$, one has

$\operatorname{Vol}\left(S^{R} \cap p_{S}^{-1}(\mathcal{B} \cap S)\right) \leq \int_{\mathcal{B} \cap S} d x \times \int_{-R}^{R}\left(1+\frac{t}{\rho}\right)^{2} d t \leq \operatorname{Area}(\mathcal{B} \cap S) \times 2 R\left(1+\frac{R^{2}}{3 \rho^{2}}\right)$.

Point a) follow from the fact that $\operatorname{Area}(\mathcal{B} \cap S)=O\left(r^{2}\right)$. We use the same kind of argument for Points b) and c).

\subsection{Stability of the Projection on a Compact Set}

Proposition 4. Let $S$ be a surface of $\mathbb{R}^{3}$ of class $C^{2}$ whose reach is greater than $\rho>0$. Let $K$ be a compact set such that $d_{H}(S, K)=\varepsilon<2 \rho$, and $R<\rho$ a positive number. If $x$ and $x^{\prime}$ are points of $S^{R}$ such that $d\left(x, x^{\prime}\right) \leq \eta$, then :

$$
\left\|p_{K}(x)-p_{K}\left(x^{\prime}\right)\right\| \leq 2 \sqrt{8 \varepsilon \rho}+2 \varepsilon+\frac{1}{1-\frac{R}{\rho}} \eta
$$

The proof of the proposition relies on Lemma 3 whose proof is given in [18].

Lemma 3. Let $S$ be a surface of $\mathbb{R}^{3}$ with a reach $\rho>0$. Let $K$ be a compact set such that $d_{H}(S, K)=\varepsilon$ with $\varepsilon \leq 2 \rho$. Let $R$ be a number such that $R<\rho$. For every $x \in S^{R}$, one has

$$
p_{K}(x) \in B\left(p_{S}(x), \sqrt{8 \varepsilon \rho}+\varepsilon\right)
$$

Proof (of Proposition 4). By the triangle inequality, we have

$$
\left\|p_{K}(x)-p_{K}\left(x^{\prime}\right)\right\| \leq\left\|p_{K}(x)-p_{S}(x)\right\|+\left\|p_{S}(x)-p_{S}\left(x^{\prime}\right)\right\|+\left\|p_{S}\left(x^{\prime}\right)-p_{K}\left(x^{\prime}\right)\right\| .
$$

It is well-known that the projection map $p_{S}$ is $\frac{1}{1-\frac{R}{\rho}}$-Lipschitz in $S^{R}$ (Theorem 4.8 of [17]). We then have $\left\|p_{S}(x)-p_{S}\left(x^{\prime}\right)\right\| \leq \frac{1}{1-\frac{R}{\rho}} \eta$. The two other terms are bounded with Lemma 3 . 


\subsection{Proof of Proposition 2}

Similarly, as for equation (11) and using the hypothesis $h \leq \frac{\rho}{2}$ and $\sqrt{8 h \rho}+$ $h \leq r$, we have $p_{Z_{h}}^{-1}(\operatorname{supp}(\chi)) \subset p_{\partial X}^{-1}\left(\mathcal{B}_{y}(2 r)\right)$. We then introduce the common set $E=\partial X^{R-h} \cap p_{\partial X}^{-1}\left(\mathcal{B}_{y}(2 r)\right)$, on which we are going to integrate. We have :

$$
\begin{aligned}
\mathcal{V}_{\partial X, R}\left(\chi_{r}\right) & =\int_{\partial X^{R} \cap p_{\partial X}^{-1}\left(\mathcal{B}_{y}(2 r)\right)}\left(x-p_{\partial X}(x)\right)\left(x-p_{\partial X}(x)\right)^{\mathbf{t}} \chi\left(p_{\partial X}(x)\right) \\
& =\int_{E}\left(x-p_{\partial X}(x)\right)\left(x-p_{\partial X}(x)\right)^{\mathbf{t}} \chi\left(p_{\partial X}(x)\right)+E r r_{1}
\end{aligned}
$$

where the error $E r r_{1}$ satisfies

$$
\left\|E r r_{1}\right\|_{\text {op }} \leq R^{2} \times\|\chi\|_{\infty} \times \operatorname{Vol}\left(\partial X^{R} \cap p_{\partial X}^{-1}\left(\mathcal{B}_{y}(2 r)\right) \backslash E\right) .
$$

Furthermore, one has $\partial X^{R} \cap p_{\partial X}^{-1}\left(\mathcal{B}_{y}(2 r)\right) \backslash E=\left[\partial X^{R} \backslash \partial X^{R-h}\right] \cap p_{\partial X}^{-1}\left(\mathcal{B}_{y}(2 r)\right)$, whose volume is bounded by Proposition 3 by $O\left(r^{2} h\right)$. Then

$$
\left\|E r r_{1}\right\|_{\text {op }}=\|\chi\|_{\infty} \times O\left(R^{2} r^{2} h\right) .
$$

Similarly, one has

$$
\mathcal{V}_{\partial X, R}\left(\chi_{r}\right)=\int_{E}\left(x-p_{Z_{h}}(x)\right)\left(x-p_{Z_{h}}(x)\right)^{\mathbf{t}} \chi\left(p_{Z_{h}}(x)\right)+E r r_{2},
$$

where the error $\operatorname{Err}_{2}$ satisfies $\left\|E r r_{2}\right\|_{\text {op }}=(R+h)^{2} \times\|\chi\|_{\infty} \times O\left(r^{2} h\right)$. We now have to compare the two integrals on the common set $E$

$\Delta=\int_{E}\left[\left(x-p_{\partial X}(x)\right)\left(x-p_{\partial X}(x)\right)^{\mathbf{t}} \chi\left(p_{\partial X}(x)\right)-\left(x-p_{Z_{h}}(x)\right)\left(x-p_{Z_{h}}(x)\right)^{\mathbf{t}} \chi\left(p_{Z_{h}}(x)\right)\right]$.

Following now the proof of Theorem 5.1 of [1], one has

$\|\Delta\|_{\text {op }} \leq\left(R^{2} \operatorname{Lip}(\chi)+2 R\left\|_{\chi}\right\|_{\infty}\right) \times[\operatorname{Vol}(E)+(\operatorname{diam}(E)+R+\sqrt{R h}) \times \operatorname{Area}(\partial E)] \times \sqrt{R h}$.

Proposition 3 gives that $\operatorname{Vol}(E)$ is bounded by $O\left(r^{2} R\right)$ and Area $(\partial E)$ is bounded by $O\left(r R+r^{2}\right)$. We then have

$$
\|\Delta\|_{\mathrm{op}}=O\left(\operatorname{Lip}(\chi) \times\left[\left(r^{3} R^{\frac{5}{2}}+r^{2} R^{\frac{7}{2}}+r R^{\frac{9}{2}}\right) h^{\frac{1}{2}}\right]+\|\chi\|_{\infty} \times\left[\left(r^{3} R^{\frac{3}{2}}+r^{2} R^{\frac{5}{2}}+r R^{\frac{7}{2}}\right) h^{\frac{1}{2}}\right]\right) .
$$

Adding the bounds of $\left\|E r r_{1}\right\|_{\text {op }},\left\|E r r_{2}\right\|_{\text {op }}$ and $\|\Delta\|_{\text {op }}$, we find the same bound :

$$
\begin{aligned}
& \left\|\mathcal{V}_{\partial X, R}\left(\chi_{r}\right)-\mathcal{V}_{Z_{h}, R}\left(\chi_{r}\right)\right\|_{\mathrm{op}} \\
& =O\left(\operatorname{Lip}(\chi) \times\left[\left(r^{3} R^{\frac{5}{2}}+r^{2} R^{\frac{7}{2}}+r R^{\frac{9}{2}}\right) h^{\frac{1}{2}}\right]+\|\chi\|_{\infty} \times\left[\left(r^{3} R^{\frac{3}{2}}+r^{2} R^{\frac{5}{2}}+r R^{\frac{7}{2}}\right) h^{\frac{1}{2}}\right]\right) .
\end{aligned}
$$

